\title{
Open-Loop Power Sharing Characteristic of a Three-Port Resonant LLC Converter
}

\author{
Yan-Kim Tran, Francisco D. Freijedo, and Drazen Dujic
}

\begin{abstract}
The Solid State Transformer (SST) is an attractive solution for highly flexible, cost-effective, compact and efficient power transfer among different grids. Furthermore, a threeport topology is proven as a suitable solution to integrate energy storage resources, the key functionality of emerging SST concept. Among other alternatives, the resonant LLC series resonant converter (SRC) is the cost-effective solution to implement the DC-transformer functionality, which is a core part of the SST. This paper addresses the power sharing characteristics and the zero-voltage switching (ZVS) conditions of a galvanically isolated three-port SRC, operated in DC-transformer mode. A mathematical model, which effectively decouples principal from circulating currents and power flows, is proposed and developed. This new mathematical framework eases the analysis; and reveals a constant power sharing characteristic tightly dominated by the resonant tank parameters even though some degrees of freedom are allowed thanks to the introduction of a differential voltage at the input terminals. Subsequently, design aspects and assessments of working operation conditions are also reported. The accuracy of the proposed model is verified by experimental validation on a labscale prototype.
\end{abstract}

Index Terms - DC-DC converters, medium-voltage DC (MVDC), multiport, resonant converter, solid state transformer.

\section{INTRODUCTION}

$\mathrm{S}_{\mathrm{n}}$ OLID State Transformer (SST) defines an emerging technology aiming for a more reliable, flexible, compact and efficient alternative to bulky line-frequency transformers (LFTs). The SST concept includes power conversion among DC and/or AC grids of a different kind [1]-[4]. The high expectations from SST solutions also include new functionalities, such as integration of energy storage, which can be made by multi-port topologies [5]-[10]. SST is already a competitive solution in several applications such as traction propulsion chain [11], [12], on-board distribution networks for marine [13], [14], photovoltaic parks [15], offshore wind-farms [16], data centers [17], or distribution in urban areas [18].

Fig. 1 shows a three-port SST solution with integrated energy storage. The overall system comprises two main kinds of subsystems: (i) DC external grids, which are interfaced by an active front-end that tightly regulate voltage/current/power at their terminals; different high level regulation strategies may be considered at this stage [1]-[4]. (ii) The DC-transformer

Manuscript received January 21, 2019.

All authors are with the École Polytechnique Fédérale de Lausanne (EPFL), Lausanne, Switzerland (e-mail: yan-kim.tran@epfl.ch).

Digital Object Identifier 10.24295/CPSSTPEA.2019.00017

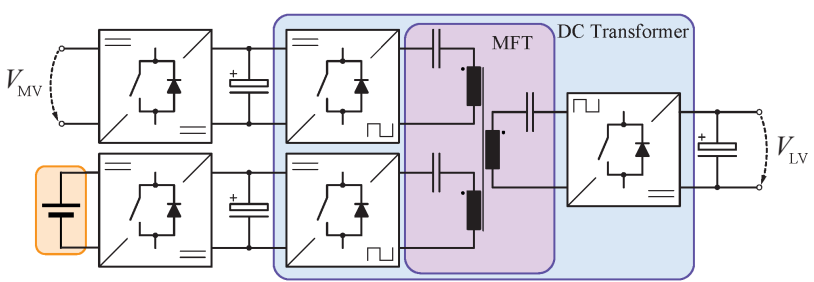

Fig. 1. A three-port SST with integrated energy storage, interfacing a medium voltage (MV) DC-grid and a low voltage (LV) DC-grid. The core of the system is a three-port resonant DC-transformer. Two active regulation stages are added in order to control the power through the DC voltage on the MV and the storage side.

operation is implemented using a three-port LLC series resonant converter; it provides voltage adaptation, natural power sharing and galvanic isolation among the different electric subsystems (in a similar manner as LFT) [4], [11], [12]. It provides a tight voltage coupling between the three ports and may, therefore, be operated in open-loop; the power conversion blocks work at a relatively high switching frequency that allows to drastically decrease the size of the so-called medium frequency transformer (MFT) [4], [11], [12], [19], [20].

Meanwhile, there is an ever increasing number of works focused on the system-level advantages of SST, the state-of-theart addressing multi-port SRC based DC-transformer contains only a few works [5]-[10]. From these works, there is an overall agreement that the resonant frequency has to be the same for all the ports, which implies a careful design of the resonant tank and parameters as well as the operation with $50 \%$ duty cycle and equal switching frequency for all the active terminals. The suitability for integration of low vottage (LV) storage elements (batteries or ultra-capacitors) is presented in [8]. Clearly, besides the feasibility of the multi-port SRC topologies, a systematic and rigorous mathematical analysis describing the natural power sharing principles is not available in the literature. The systematic evaluation is needed to address the limits of the topology and derive design rules that permit to consider the technology at an industry level. And even though the modelling with the well known First Harmonic Approximation (FHA) [6], [7] is possible, the detailed description of the waveforms, required for the soft switching and the losses evaluation is not captured by this method.

To cover the lack of analysis that may help in the elaboration of design rules, this paper presents a rigorous mathematical modeling of the three-port LLC-based DC transformer; the natural power sharing, as a function of the MFT parameters, is derived and characterized. To do so, an equivalent model that simplifies significantly the original mathematical model of the 
multi-port DC transformer, but still enables the precision required for the soft switching characterization, is proposed. Subsequently, it is discussed how the natural power sharing can be modified by acting on the input voltages; the influence of the source voltages is accurately quantified using the proposed model. This part of the analysis gives an idea of how effective is SST regulation at an interface level (point i) of the operation described above). The conditions that lead to a loss of zero-voltage switching (ZVS) operation, and hence to a potential efficiency drop, are also identified from the equivalent model provided. Comprehensive experimental tests fully verify the theoretical modelling and, therefore, validate the proposed design insights.

The organization of the rest of the paper is summarized as follows. The next section presents the problem description and the modelling challenges to provide self-contained design guidelines. Section III shows the proposed mathematical approach, which is based on a change of variable that allows obtaining two equivalent decoupled systems. Section IV derives the power sharing characteristics as a function of the MFT parameters, input-voltages and losses. Section V shows the ZVS regions for the DC-transformer operation. Section VI shows and discusses the experimental results. Finally, the conclusion summarizes the most important findings.

\section{Circuit and Problem Description}

While all three ports are made from active switching elements, they can be operated with active switching actions or be used as passive diode rectifier, depending on the power flow. The mode of operation analyzed in details in this paper, considering the DC-transformer with two inputs and one output, is depicted in Fig. 2. The circuit includes a three-winding MFT with the number of turns given by $n_{1}, n_{2}, n_{3}$. The input ports ( 1 and 2) are active sources equipped with two resonant tanks of the identical resonant frequency $f_{\text {res }}$ composed by the capacitors $C_{1}$ and $C_{2}{ }^{\prime}$ combined with the leakage inductance $L_{1}$ and $L_{2}^{\prime}$. The third port acts as a passive load. The semiconductors of the input ports are switched at the same fixed frequency with a constant duty-cycle of $50 \%$ and the same phase [10]. In order to benefit not only from ZVS [21] and reduced turn-off current on the primary switches but also from zero-current switching (ZCS) on the secondary diode rectifier, the switching frequency is set slightly below the resonant frequency $\left(f_{\mathrm{sw}}<f_{\text {res }}\right)$ [22]. The DC bus capacitors $C_{\mathrm{DC}}$ are sized much bigger than the resonant capacitors, so, for the purpose of modelling their voltage $V_{\mathrm{DC}}$ can be considered constant; i.e., square-wave voltage sources of amplitude $V_{1}=V_{\mathrm{CD}_{1}} / 2$ and $V_{2}^{\prime}=V_{\mathrm{CD}_{2}}^{\prime} / 2$ are considered. The power transferred to the load port $\left(P_{\text {load }}=P_{3}\right)$ is the sum of both contributions $P_{1}$ and $P_{2}$ from each active ports.

The converter is sized to be operated in a half-cycle discontinuous mode (HC-DCM) [23] over its complete operating range [10]. Thus two intervals can be identified per half-period, as depicted in Fig. 3 and detailed in the following. It may also be noticed that, for modelling purposes, the circuit parameters $L_{2}{ }^{\prime}, C_{2}^{\prime}, V_{2}^{\prime}$ and $V_{3}^{\prime}$ are referred to the port 1 of the transformer and represented by $L_{2}=L_{2}^{\prime} n_{1}^{2} / n_{2}^{2}, C_{2}=C_{2}^{\prime} n_{2}^{2} / n_{1}^{2}$, $V_{2}=V_{2}^{\prime} n_{1} / n_{2}$ and $V_{3}=V_{3}^{\prime} n_{1} / n_{3}$ (for the sake of simplicity,

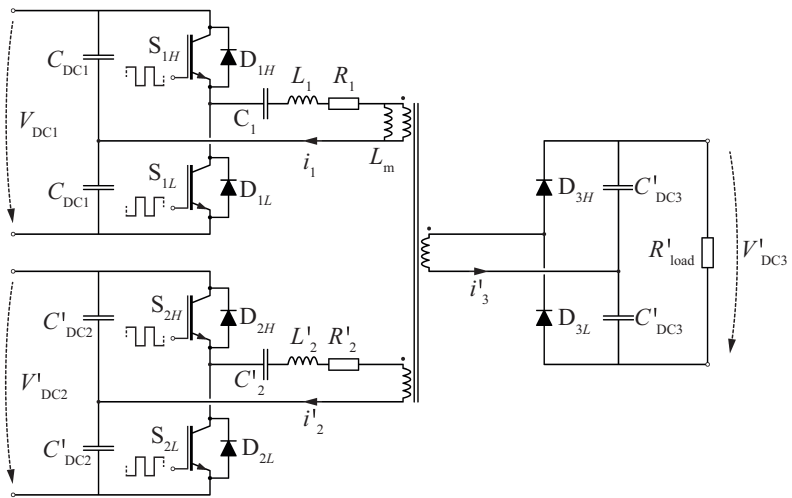

Fig. 2. Topology of a three-port resonant DC-DC converter working as DC transformer (two actively switched ports and one passive load).

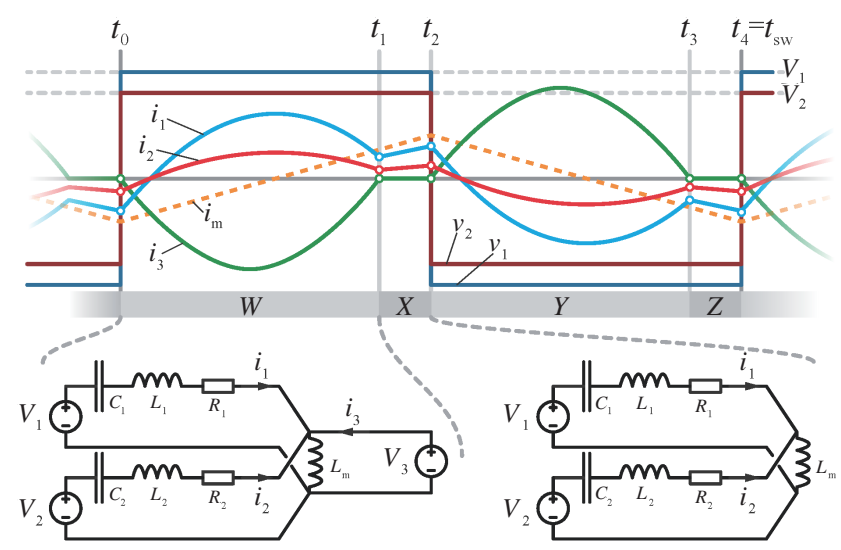

Fig. 3. Equivalent circuits and steady state voltage and current waveforms during the two different intervals of three-port DC transformer operation.

a loss-less converter without parasitic resistances, i.e., $R_{1}=$ $R_{2}=0$, is considered firstly). During the interval $W$, the two active ports are delivering power to the load as well as the magnetizing current $i_{\mathrm{m}}$; the voltage seen at the magnetizing inductance terminals is clamped by the voltage on the load port due to the conduction of the diodes [9], [10]. When the output diode rectifier stops conducing, interval $X$ starts. The two active ports are only supporting $i_{\mathrm{m}}$, and the magnetizing inductance terminals are no longer clamped, while the load is supported by the output capacitors. Intervals $Y$ and $Z$ are the same as $W$ and $X$ respectively with opposite voltage and current polarity. The circuit time-domain equations should be calculated for each interval. For the interval $W$, the two input $(k=1,2)$ voltages, and currents are given respectively by:

$$
\begin{aligned}
& v_{k}(t)=v_{C_{k}}^{W}(t)+L_{k} \frac{\mathrm{d} i_{k}^{W}(t)}{\mathrm{d} t}+v_{3}(t) \\
& i_{k}(t)=C_{k} \frac{\mathrm{d} v_{C_{k}}^{W}(t)}{\mathrm{d} t} .
\end{aligned}
$$

The voltage and current of the third port (output) are given by:

$$
\begin{aligned}
& v_{3}(t)=L_{\mathrm{m}} \frac{\mathrm{d} i_{\mathrm{m}}^{W}(t)}{\mathrm{d} t} \\
& i_{3}^{W}(t)=-i_{1}^{W}(t)-i_{2}^{W}(t)+i_{\mathrm{m}}^{W}(t) .
\end{aligned}
$$


The Laplace transform with initial conditions is considered to obtain time-domain expressions. From the step response at $t=0$, and assuming ideal voltage sources, of amplitudes $V_{1}, V_{2}$ and $V_{3}$, respectively:

$$
\begin{aligned}
& \frac{V_{1}}{s}=\frac{v_{C_{1}}\left(t_{0}\right)}{s}+\frac{1}{s C_{1}} i_{1}^{W}(s)+s L_{1} i_{1}^{W}(s)-L_{1} i_{1}\left(t_{0}\right)+\frac{V_{3}}{s} \\
& \frac{V_{2}}{s}=\frac{v_{C_{2}}\left(t_{0}\right)}{s}+\frac{1}{s C_{2}} i_{2}^{W}(s)+s L_{2} i_{2}^{W}(s)-L_{2} i_{2}\left(t_{0}\right)+\frac{V_{3}}{s} \\
& i_{\mathrm{m}}^{W}(s)=\frac{V_{3}}{s^{2} L_{\mathrm{m}}}+\frac{i_{1}\left(t_{0}\right)+i_{2}\left(t_{0}\right)}{s} .
\end{aligned}
$$

Expressions for $i_{1}(s)$ and $i_{2}(s)$ can be simplified by introducing $\omega^{2}=1 /\left(L_{1} C_{1}\right)=1 /\left(L_{2} C_{2}\right)$, which gives (for $k=1,2$ ):

$$
i_{k}^{W}(s)=\left(\frac{V_{k}-v_{C_{k}}\left(t_{0}\right)-V_{3}}{\omega L_{k}}\right) \frac{\omega}{\omega^{2}+s^{2}}+i_{k}\left(t_{0}\right) \frac{s}{\omega^{2}+s^{2}}
$$

Back in time domain, it yields:

$$
i_{k}^{W}(t)=i_{k}\left(t_{0}\right) \cos (\omega t)+\left(\frac{V_{k}-v_{C_{k}}\left(t_{0}\right)-V_{3}}{\omega L_{k}}\right) \sin (\omega t)
$$

and

$$
i_{\mathrm{m}}^{W}(t)=i_{1}\left(t_{0}\right)+i_{2}\left(t_{0}\right)+\frac{V_{3}}{L_{\mathrm{m}}} t
$$

For the interval $X$, the voltage equations are:

$$
\begin{aligned}
& V_{1}=v_{C_{1}}^{X}(t)+L_{1} \frac{\mathrm{d} i_{1}^{X}(t)}{\mathrm{d} t}+L_{\mathrm{m}} \frac{\mathrm{d}\left(i_{1}^{X}(t)+i_{2}^{X}(t)\right)}{\mathrm{d} t} \\
& V_{2}=v_{C_{2}}^{X}(t)+L_{2} \frac{\mathrm{d} i_{2}^{X}(t)}{\mathrm{d} t}+L_{\mathrm{m}} \frac{\mathrm{d}\left(i_{1}^{X}(t)+i_{2}^{X}(t)\right)}{\mathrm{d} t} .
\end{aligned}
$$

Using the Laplace transform and the initial conditions $i_{1}\left(t_{1}\right)$, $i_{2}\left(t_{1}\right), v_{C_{1}}\left(t_{1}\right)$ and $v_{C_{2}}\left(t_{1}\right)$ :

$$
\begin{aligned}
& \frac{V_{1}}{s}=\frac{v_{C_{1}}\left(t_{1}\right)}{s}+\frac{1}{s C_{1}} i_{1}^{X}(s)+s L_{1} i_{1}^{X}(s)-L_{1} i_{1}\left(t_{1}\right)+v_{\mathrm{m}}^{X}(s) \\
& \frac{V_{2}}{s}=\frac{v_{C_{2}}\left(t_{1}\right)}{s}+\frac{1}{s C_{2}} i_{2}^{X}(s)+s L_{2} i_{2}^{X}(s)-L_{2} i_{2}\left(t_{1}\right)+v_{\mathrm{m}}^{X}(s) .
\end{aligned}
$$

And subsequently, it yields:

$$
\begin{aligned}
& i_{1}^{X}(s)=\left(\frac{V_{1}}{s}-\frac{v_{C_{1}}\left(t_{1}\right)}{s}+L_{1} i_{1}\left(t_{1}\right)-v_{\mathrm{m}}^{X}(s)\right) \frac{1}{\frac{1}{s C_{1}}+s L_{1}} \\
& i_{2}^{X}(s)=\left(\frac{V_{2}}{s}-\frac{v_{C_{2}}\left(t_{1}\right)}{s}+L_{2} i_{2}\left(t_{1}\right)-v_{\mathrm{m}}^{X}(s)\right) \frac{1}{\frac{1}{s C_{2}}+s L_{2}} \\
& v_{\mathrm{m}}^{X}(s)=s L_{\mathrm{m}}\left(i_{1}(s)+i_{2}(s)\right)-L_{\mathrm{m}}\left(i_{1}\left(t_{1}\right)+i_{2}\left(t_{1}\right)\right)
\end{aligned}
$$

Solving the system given by the equations (9), the three currents in the interval $X$ become:

$$
\begin{aligned}
i_{1}^{X}(t)= & A \cos (\omega t)+B \sin (\omega t) \\
& +\frac{L_{2}}{L_{1}+L_{2}}\left(C \cos \left(\omega_{X} t\right)+D \sin \left(\omega_{X} t\right)\right) \\
i_{2}^{X}(t)= & -A \cos (\omega t)-B \sin (\omega t) \\
& +\frac{L_{1}}{L_{1}+L_{2}}\left(C \cos \left(\omega_{X} t\right)+D \sin \left(\omega_{X} t\right)\right) \\
i_{\mathrm{m}}(t)= & C \cos \left(\omega_{X} t\right)+D \sin \left(\omega_{X} t\right)
\end{aligned}
$$

with the constants being equal to:

$$
\begin{aligned}
A & =\frac{L_{1}}{L_{1}+L_{2}} i_{1}\left(t_{1}\right)-\frac{L_{2}}{L_{1}+L_{2}} i_{2}\left(t_{1}\right) \\
B & =\frac{V_{1}-V_{2}-v_{C_{1}}\left(t_{1}\right)+v_{C_{2}}\left(t_{1}\right)}{\omega\left(L_{1}+L_{2}\right)} \\
C & =i_{1}\left(t_{1}\right)+i_{2}\left(t_{1}\right) \\
D & =\frac{L_{2}\left(V_{1}-v_{C_{1}}\left(t_{1}\right)\right)+L_{1}\left(V_{2}-v_{C_{2}}\left(t_{1}\right)\right)}{\omega_{X}\left(L_{1} L_{2}+L_{1} L_{\mathrm{m}}+L_{2} L_{\mathrm{m}}\right)} \\
\omega_{X} & =\sqrt{\frac{1}{\left(\frac{L_{1} L_{2}}{L_{1}+L_{2}}+L_{\mathrm{m}}\right)\left(C_{1}+C_{2}\right)}} .
\end{aligned}
$$

By inspection of (10) and (11), the two input subsystems are strongly coupled during the $X$ interval. Eventually, in the search for insightful design guidelines, dealing with a high order highly coupled model has been found forbiddingly complex. Alternatively, a change of variable taking into account the topology is proposed, as detailed in the next section.

\section{Proposed ModeL}

A systematic analytic development, based on changes of variable, which permits to derive power sharing rules is provided in this section. The idea is to obtain a set of variables that are decoupled during all the intervals. Looking at the parallel/series symmetries in Fig. 2, the following substitutions in (5) and (10) are proposed. A common mode equivalent or parallel combination of the input ports is identified. The input voltage and current become

$$
\begin{aligned}
& v_{\mathrm{P}}(t)=\frac{L_{2} v_{1}(t)+L_{1} v_{2}(t)}{L_{1}+L_{2}} \\
& i_{\mathrm{P}}(t)=i_{1}(t)+i_{2}(t)
\end{aligned}
$$

while the parallel equivalent resonant tank is given by

$$
\begin{aligned}
& L_{\mathrm{P}}=\frac{L_{1} L_{2}}{L_{1}+L_{2}} \\
& C_{\mathrm{P}}=C_{1}+C_{2} .
\end{aligned}
$$

Then, the differential mode equivalent is characterized by the voltage and current expressed as 


$$
\begin{aligned}
v_{\mathrm{S}}(t) & =v_{1}(t)-v_{2}(t) \\
i_{\mathrm{S}}(t) & =\frac{L_{1}}{L_{1}+L_{2}} i_{1}(t)-\frac{L_{2}}{L_{1}+L_{2}} i_{2}(t) .
\end{aligned}
$$

The series combination of the resonant tank gives

$$
\begin{aligned}
& L_{\mathrm{S}}=L_{1}+L_{2} \\
& C_{\mathrm{S}}=\frac{C_{1} C_{2}}{C_{1}+C_{2}} .
\end{aligned}
$$

The "P" and "S" subscripts refer to parallel and series equivalents, but may be also related to common and differential modes. After substituting original variables by "P" and "S" equivalents, the interval $W$ circuit is described by

$$
\begin{gathered}
i_{\mathrm{S}}^{W}(t)=i_{\mathrm{S}}\left(t_{0}\right) \cos (\omega t)+\left(\frac{V_{\mathrm{S}}-v_{C \mathrm{~S}}\left(t_{0}\right)}{\omega L_{\mathrm{S}}}\right) \sin (\omega t) \\
i_{\mathrm{P}}^{W}(t)=i_{\mathrm{P}}\left(t_{0}\right) \cos (\omega t)+\left(\frac{V_{P}-v_{C_{\mathrm{P}}}\left(t_{0}\right)-V_{3}}{\omega L_{\mathrm{P}}}\right) \sin (\omega t) .
\end{gathered}
$$

Similarly, for the interval $X$

$$
\begin{aligned}
& i_{\mathrm{S}}^{X}(t)=i_{\mathrm{S}}\left(t_{1}\right) \cos (\omega t)+\left(\frac{V_{\mathrm{S}}-v_{C_{\mathrm{S}}}\left(t_{1}\right)}{\omega L_{\mathrm{S}}}\right) \sin (\omega t) \\
& i_{\mathrm{P}}^{X}(t)=i_{\mathrm{P}}\left(t_{1}\right) \cos \left(\omega_{X} t\right)+\left(\frac{V_{\mathrm{P}}-v_{C_{\mathrm{P}}}\left(t_{1}\right)}{\omega_{X}\left(L_{\mathrm{P}}+L_{\mathrm{m}}\right)}\right) \sin \left(\omega_{X} t\right) .
\end{aligned}
$$

It is worth noting that $i_{\mathrm{S}}^{W}(t)$ and $i_{\mathrm{S}}^{X}(t)$ can be merged in

$$
i_{\mathrm{S}}(t)=i_{\mathrm{S}}\left(t_{0}\right) \cos (\omega t)+\left(\frac{V_{\mathrm{S}}-v_{C_{\mathrm{S}}}\left(t_{0}\right)}{\omega L_{\mathrm{S}}}\right) \sin (\omega t)
$$

which holds valid for all intervals defined by $\left(0, t_{\mathrm{sw}} / 2\right)$.

After the transformation, two fully decoupled "P" and equivalent systems are obtained. The sub-circuits correspond to each circuit and interval are depicted in Figs. 4 and 5. By inspection of Figs. 4 and 5, the power delivery to the load depends on the "P" circuit, meanwhile the "S" circuit represents power re-circulation between the sources.

\section{Power Sharing Expressions}

As early mentioned, the ideal loss-less case is considered firstly (in order to ease the mathematical development). However, the losses are also included in subsection IV-B, since they have a relevant role in the power sharing characteristics.

\section{A. Ideal Case}

The power delivered to the load is modelled in the "P" circuit (cf., Fig. 4), which is defined by

$$
\left\langle P_{\mathrm{P}}>=\frac{1}{t_{\mathrm{sw}}} \int_{0}^{t_{\mathrm{s}}} v_{\mathrm{P}}(t) i_{\mathrm{P}}(t) \mathrm{d} t=\frac{2 V_{\mathrm{P}}}{t_{\mathrm{sw}}} \int_{0}^{t_{\mathrm{sw}} / 2} i_{\mathrm{p}}(t) \mathrm{d} t\right.
$$

It should be noticed that "S" circuit is purely reactive (cf.,

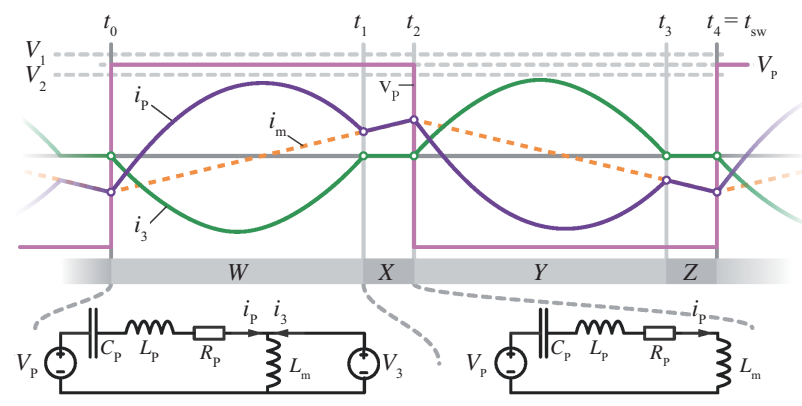

Fig. 4. "P" equivalent intervals and sub-circuits (common mode power component).

Fig. 5), so

$$
\int_{0}^{t_{\mathrm{m}} / 2} i_{\mathrm{S}}(t) \mathrm{d} t=0
$$

The average power transferred to the load by each active port $(k=1,2)$ is given by

$$
\left\langle P_{k}>=\frac{2}{t_{\mathrm{sw}}} \int_{0}^{t_{\mathrm{sw}} / 2} v_{k}(t) i_{k}(t) \mathrm{d} t=\frac{2 V_{k}}{t_{\mathrm{sw}}} \int_{0}^{t_{\mathrm{sw}} / 2} i_{k}(t) \mathrm{d} t .\right.
$$

Development of this expression for the port 1, and also having into account (22) and the changes of variable in (12) to (15), gives

$$
\begin{aligned}
<P_{1}>= & \frac{L_{2}}{L_{1}+L_{2}} \frac{2 V_{\mathrm{P}}}{t_{\mathrm{sw}}} \int_{0}^{t_{\mathrm{s}} / 2} i_{\mathrm{P}}(t) \mathrm{d} t \\
& +\frac{2 V_{\mathrm{S}} L_{1} L_{2}}{t_{\mathrm{sw}}\left(L_{1}+L_{2}\right)^{2}} \int_{0}^{t_{\mathrm{sw}} / 2} i_{\mathrm{P}}(t) \mathrm{d} t \\
= & \frac{L_{2}}{L_{1}+L_{2}}<P_{\mathrm{P}}>+\frac{L_{1} L_{2}}{\left(L_{1}+L_{2}\right)^{2}} \frac{V_{\mathrm{S}}}{V_{\mathrm{P}}}<P_{\mathrm{P}}>.
\end{aligned}
$$

And, for the second port

$$
<P_{2}>=\frac{L_{1}}{L_{1}+L_{2}}<P_{\mathrm{P}}>-\frac{L_{1} L_{2}}{\left(L_{1}+L_{2}\right)^{2}} \frac{V_{\mathrm{S}}}{V_{\mathrm{P}}}<P_{\mathrm{P}}>.
$$

By inspection of (24) and (25), in a rated condition, the part of the total power that corresponds to each port is a function of the MFT leakage inductances. A deviation from the ratio is caused by the presence of a differential voltage $V_{\mathrm{S}}$ (which may be regulated outside the resonant multi-port stage), but will, in practice, remain rather small as $V_{\mathrm{S}}<<V_{\mathrm{P}}$ is expected.

At this point, it is key to stress that the "P" and " $\mathrm{S}$ " equivalents permit to calculate the power sharing rules without needing to solve the initial conditions of the circuit, which drastically eases the analysis and reveals design rules insights based on natural power sharing characteristics.

Anyway, as the equivalent circuits "P" and "S" are fully decoupled, the steady-state boundary conditions may be calculated solving: 


$$
\begin{gathered}
i_{\mathrm{P}}^{W}\left(t_{0}\right)=-i_{\mathrm{P}}^{X}\left(t_{2}\right) \\
v_{C_{\mathrm{p}}}^{W}\left(t_{0}\right)=-v_{C_{\mathrm{p}}}^{X}\left(t_{2}\right) \\
i_{\mathrm{S}}^{W}\left(t_{0}\right)=-i_{\mathrm{S}}^{X}\left(t_{2}\right) \\
v_{C_{\mathrm{S}}}^{W}\left(t_{0}\right)=-v_{C_{\mathrm{S}}}^{X}\left(t_{2}\right) .
\end{gathered}
$$

These expressions are employed to assess the ZVS conditions, in Section V.

\section{1) Worst Case Analysis for Tolerances of the Resonant Tank} Parameters

If tolerance values are considered for the resonant tank parameters, the assumption of an identical resonant frequency for both resonant tanks, used to get (4), is no longer valid and $\omega_{1}=\sqrt{1 / L_{1} C_{1}} \neq \omega_{2}=\sqrt{1 / L_{2} C_{2}}$. The expressions for power sharing becomes:

$$
\begin{aligned}
<P_{1}>= & \frac{L_{2} \omega_{2}}{L_{1} \omega_{1}+L_{2} \omega_{2}}<P_{\mathrm{P}}> \\
& +\frac{L_{1} L_{2} \omega_{1} \omega_{2}}{\left(L_{1} \omega_{1}+L_{2} \omega_{2}\right)^{2}} \frac{V_{\mathrm{S}}}{V_{\mathrm{P}}}<P_{\mathrm{P}}>.
\end{aligned}
$$

And, for the second port

$$
\begin{aligned}
<P_{2}>= & \frac{L_{1} \omega_{1}}{L_{1} \omega_{1}+L_{2} \omega_{2}}<P_{\mathrm{P}}> \\
& -\frac{L_{1} L_{2} \omega_{1} \omega_{2}}{\left(L_{1} \omega_{1}+L_{2} \omega_{2}\right)^{2}} \frac{V_{S}}{V_{\mathrm{P}}}<P_{P}>.
\end{aligned}
$$

Assuming $V_{\mathrm{S}}=0$,

$$
<P_{1}>=\frac{\sqrt{L_{2} / C_{2}}}{\sqrt{L_{2} / C_{2}}+\sqrt{L_{1} / C_{1}}}<P_{\mathrm{P}}>.
$$

And, for the second port

$$
<P_{2}>=\frac{\sqrt{L_{1} / C_{1}}}{\sqrt{L_{1} / C_{1}}+\sqrt{L_{2} / C_{2}}}<P_{\mathrm{P}}>.
$$

The worst case scenarios correspond to the values that maximize/minimize $\left\langle P_{1}\right\rangle$ and $\left\langle P_{2}\right\rangle$; by inspection of (29) and (30) these correspond to the combinations of i) ( $L_{1}^{\min }, C_{1}^{\max }$, $\left.L_{2}^{\max }, C_{2}^{\min }\right)$ and ii) $\left(L_{1}^{\max }, C_{1}^{\min }, L_{2}^{\min }, C_{2}^{\max }\right)$.

\section{B. Consideration of System Losses}

The circuit losses during operation, such as conduction and switching ones, are reflected on the system dynamics and, therefore, can be modeled by equivalent resistors [24]. An a priory estimation of their values is a complex task, but they can be measured from the experimental curves [24]. Equivalent series resistors per port (i.e., $R_{1}$ and $R_{2}$ ) are included in the

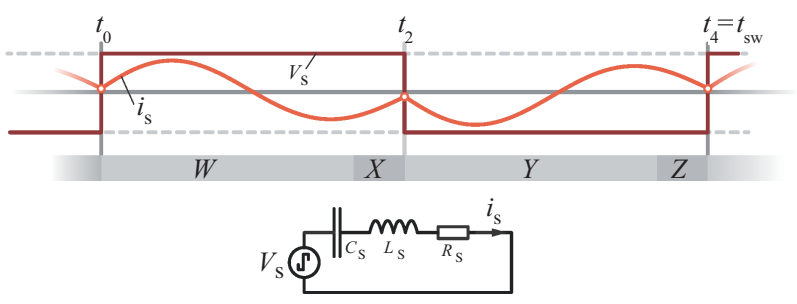

Fig. 5. "S" equivalent intervals and sub-circuits (differential mode power component).

analysis, with $R_{1} / L_{1} \approx R_{2} / L_{2}$ being assumed. Subsequently, the "P" and "S" equivalents are defined as

$$
R_{\mathrm{P}}=\frac{R_{1} R_{2}}{R_{1}+R_{2}} \text { and } R_{\mathrm{S}}=R_{1}+R_{2} .
$$

Figs. 4 and 5 already include these terms. (23) is re-calculated considering the voltage drops at $R_{\mathrm{P}}$ and $R_{\mathrm{S}}$ and also taking into account that (22) is no longer true (i.e., " $\mathrm{S}$ " circuit is not purely reactive) and gives:

$$
\begin{aligned}
<P_{1}>= & \frac{L_{2}}{L_{1}+L_{2}}<P_{\mathrm{P}}>+\frac{L_{1} L_{2}}{\left(L_{1}+L_{2}\right)^{2}} \frac{V_{\mathrm{S}}}{V_{\mathrm{P}}}<P_{\mathrm{P}}> \\
& +<P_{\mathrm{S}}>+\frac{L_{2}}{L_{1}+L_{2}}<P_{\mathrm{P}_{\text {loss }}}>+\frac{L_{1}}{L_{1}+L_{2}}<P_{\mathrm{S}_{\text {loss }}}> \\
<P_{2}>= & \frac{L_{1}}{L_{1}+L_{2}}<P_{\mathrm{P}}>-\frac{L_{1} L_{2}}{\left(L_{1}+L_{2}\right)^{2}} \frac{V_{\mathrm{S}}}{V_{\mathrm{P}}}<P_{\mathrm{P}}> \\
& -<P_{\mathrm{S}}>+\frac{L_{1}}{L_{1}+L_{2}}<P_{\mathrm{P}_{\text {loos }}}>+\frac{L_{2}}{L_{1}+L_{2}}<P_{\mathrm{S}_{\text {loss }}}>
\end{aligned}
$$

The losses are included within the new terms in (32):

$$
\begin{aligned}
& <P_{\mathrm{P}_{\mathrm{loss}}}>=\frac{R_{\mathrm{P}}}{t_{\mathrm{sw}}} \int_{0}^{t_{\mathrm{sw}}} i_{\mathrm{P}}^{2}(t) \mathrm{d} t \\
& <P_{\mathrm{S}_{\mathrm{loss}}}>=\frac{R_{\mathrm{S}}}{t_{\mathrm{sw}}} \int_{0}^{t_{\mathrm{sw}}} i_{\mathrm{S}}^{2}(t) \mathrm{d} t \\
& <P_{\mathrm{S}}>=V_{\mathrm{P}} \frac{2}{t_{\mathrm{sw}}} \int_{0}^{t_{\mathrm{sw}} / 2} i_{\mathrm{S}}(t) \mathrm{d} t .
\end{aligned}
$$

It has to be noted that the active component of the differential current $i_{\mathrm{S}}(t)$ is due to the presence of $R_{\mathrm{S}}$. In order to simplify the notation, the factor $k_{12}$ and $k_{21}$ are introduced and are given by:

$$
\begin{aligned}
& k_{12}=\frac{L_{2}}{L_{1}+L_{2}} \\
& k_{21}=\frac{L_{1}}{L_{1}+L_{2}}
\end{aligned}
$$

Since $k_{12}$ and $k_{21}$ are smaller than 1 and $V_{\mathrm{S}}<<V_{\mathrm{P}}$, the second term of (32) becomes very small and can be neglected. If the same is applied to the losses, which are also expected very small, (32) can be simplified in (35) which leads to the graphical representation of Fig. 6. 


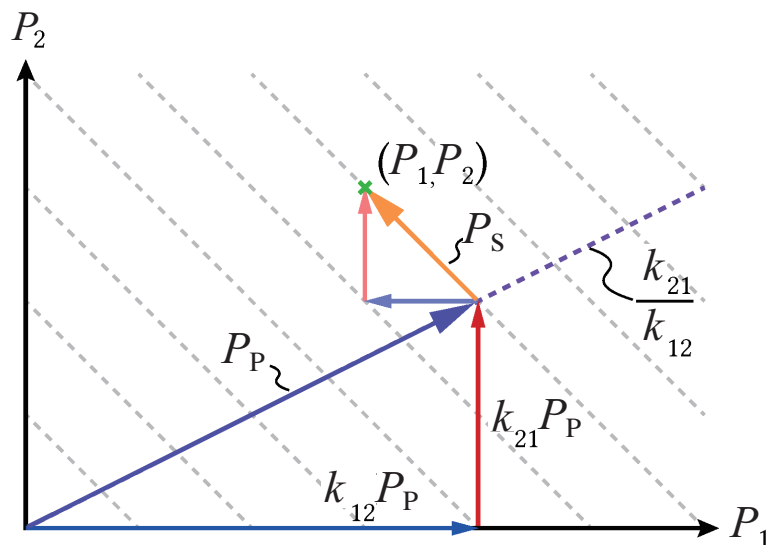

Fig. 6. Graphical representation of $P_{1}$ and $P_{2}$ in function of $P_{\mathrm{P}}$ and $P_{\mathrm{S}}$. The axis where $P_{\mathrm{S}}=0$ is defined by the resonant tank design with $k_{12}$ and $k_{21}$.

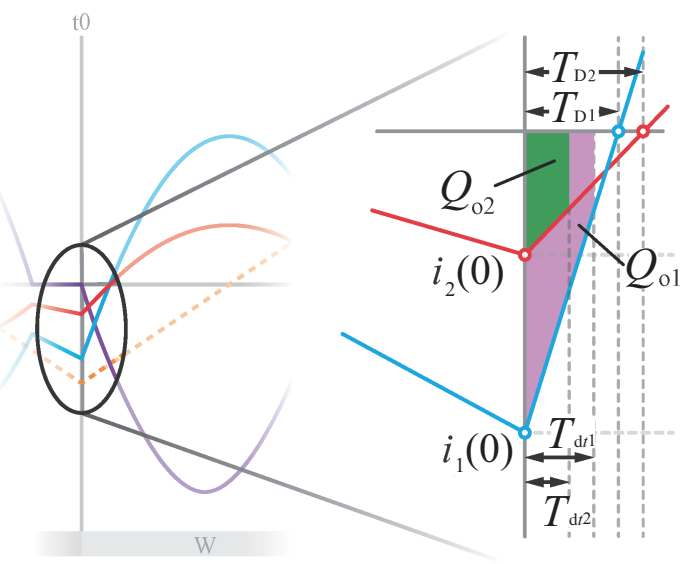

Fig. 7. Variables that set ZVS conditions.

$$
\begin{aligned}
& <P_{1}>\simeq k_{12}<P_{\mathrm{P}}>+<P_{\mathrm{S}}> \\
& <P_{2}>\simeq k_{21}<P_{\mathrm{P}}>-<P_{\mathrm{S}}>
\end{aligned}
$$

\section{Assessment OF ZVS OpERATION}

ZVS operation is defined electrically by a set of conditions described in Fig. 7. First, during the turn-on event of a semiconductor, the dead time $T_{\mathrm{d} t, k}$ (adequately applied for the selected semiconductor voltage class) must be shorter than $T_{\mathrm{D}, k}$ (according to Fig. 7, this interval starts with a load switching event and ends when $i_{k}(t)$ crosses zero): i.e,

$$
T_{\mathrm{d}, k}<T_{\mathrm{D}, k} \quad \text { for } k=1,2 .
$$

Second, the current flowing through the switch during $T_{\mathrm{d} t, k}$ must be large enough to charge the output capacitance $C_{\text {oes, } k}$ (available from semiconductor data-sheet): from 0 to $V_{\mathrm{dc}, k}$ during the turning off; from $V_{\mathrm{dc}, k}$ to 0 during the turning on. This condition can be mathematically described by

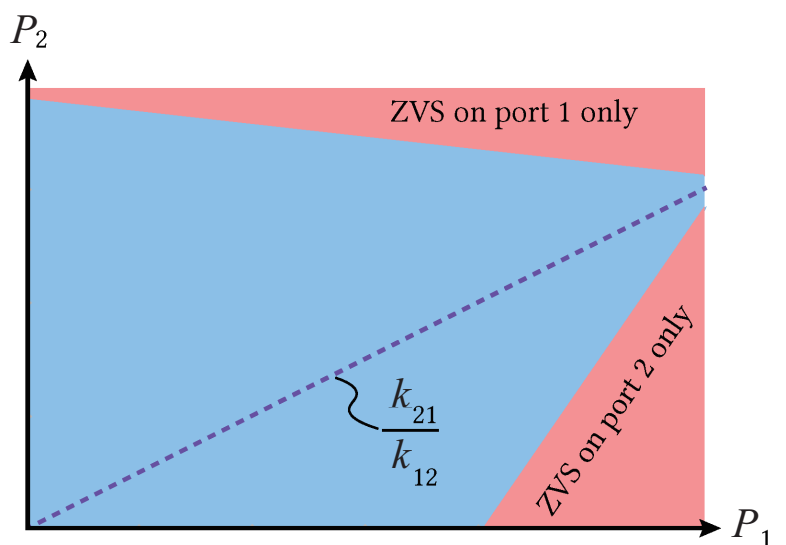

Fig. 8. Theoretical ZVS operation area. It shows that the ZVS region is well impacted by the design of the resonant tank, with $k_{21} / k_{12}$.

$$
Q_{\mathrm{o}, k}=\int_{0}^{T_{\mathrm{d} t, k}} i_{k}(t) \mathrm{d} t>2 V_{\mathrm{dc}, k} C_{\mathrm{oes}, k} \quad \text { for } k=1,2
$$

with $Q_{\mathrm{o}, k}$ being the integral of current during $T_{\mathrm{d} t, k \text {. For a given }}$ operation point, $Q_{\mathrm{o}, k}$ can be approximated by the area of the trapeze defined by:

$$
Q_{\mathrm{o}, k}=\frac{T_{\mathrm{d} t, k} i_{k}(0)}{2}\left(1+\frac{T_{\mathrm{d} t, k}}{T_{\mathrm{D}, k}}\right)
$$

Currents $i_{1}(t)$ and $i_{2}(t)$ are needed to assess ZVS conditions at any operation point. The use of "P" and "S" equivalents helps in this assessment. First the original variables $i_{1}$ and $i_{2}$ are considered with the inverse of (12) and (14).

$$
\begin{aligned}
& i_{1}(t)=i_{\mathrm{S}}(t)+\frac{L_{2}}{L_{1}+L_{2}} i_{\mathrm{P}}(t) \\
& i_{2}(t)=-i_{\mathrm{S}}(t)+\frac{L_{1}}{L_{1}+L_{2}} i_{\mathrm{P}}(t) .
\end{aligned}
$$

The initial conditions of $i_{P}(t)$ may be evaluated in function of $P_{\mathrm{P}}$ as well as $i_{\mathrm{S}}(t)$ is expressed in function of $P_{\mathrm{S}}$ and the ZVS conditions may be verified over the complete operation plane defined by $P_{1}$ and $P_{2}$. Applying bi-port LLC design rules [25] to the "P" circuit, ZVS can be ensured on the axis given by $k_{21} / k_{12}$. By inspection of Fig. 7, Fig. 6 and (39), it can be noticed that the presence of increasing $i_{\mathrm{S}}$ tends to push one of the subsystems out of the ZVS condition. This is illustrated in Fig. 8 which reveals the ZVS region over the plane $\left(P_{1}, P_{2}\right)$. It can be drawn that by sizing the resonant tank $\left(L_{1}\right.$ and $\left.L_{2}\right)$ adequately, the ZVS region can be influenced in order to match a specific operation region.

\section{EXPERIMENTAL RESULTS}

To demonstrate the validity of the developed model and power sharing in practice, a $4 \mathrm{~kW}$ rated converter comprising a three-winding transformer with a 1:1:1 turn ratio is realized. Fig. 9 shows a photograph of the lab-scale prototype. The 


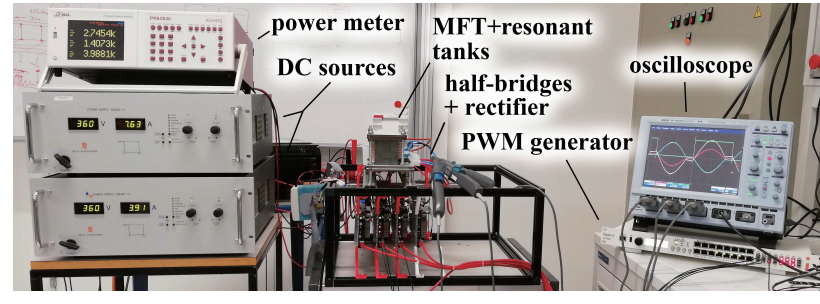

Fig. $9.4 \mathrm{~kW} / 360 \mathrm{~V}$ rated experimental test-setup.

TABLE I

Resonant TANK Parameters

\begin{tabular}{cccccc}
\hline \hline$L_{\mathrm{m}}$ & $L_{1}$ & $C_{1}$ & $L_{2}$ & $C_{2}$ & $f_{\text {res }}$ \\
\hline $400 \mu \mathrm{H}$ & $17.5 \mu \mathrm{H}$ & $5 \mu \mathrm{F}$ & $35 \mu \mathrm{H}$ & $2.5 \mu \mathrm{F}$ & $17 \mathrm{kHz}$ \\
\hline \hline
\end{tabular}

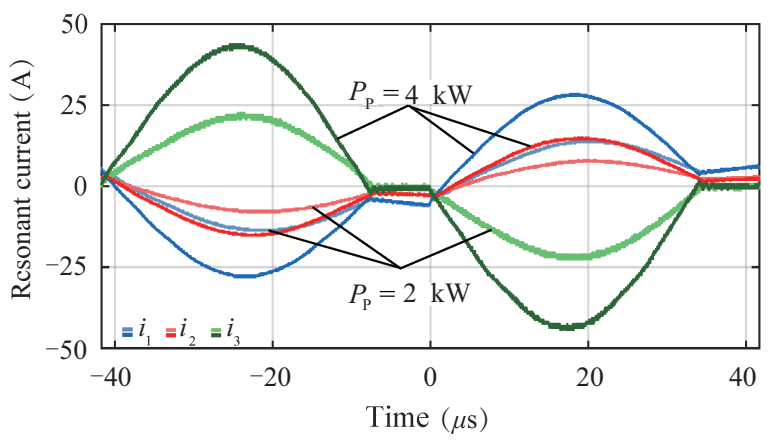

Fig. 10. Resonant current waveforms (oscilloscope import) from measurement at $2 \mathrm{~kW}$ (light colors) and $4 \mathrm{~kW}$ (dark colors).

MFT is designed to be operated at $f_{\mathrm{sw}}=12 \mathrm{kHz}$. The load port has a leakage inductance around $0.8 \mu \mathrm{H}$, and the two active ports are equipped with resonant tanks with the parameters presented in Table I. Assuming a 5\% tolerance for the resonant tank components in Table I, the maximum deviations for $P_{1}$ and $P_{2}$ are $6.8 \%$ and $3.4 \%$, respectively. The voltages $V_{1}$ and $V_{2}$ are generated with two DC power supplies, allowing to vary precisely the voltage of the two DC buses and test various operating points around $360 \mathrm{~V}$. The three half-bridge converters are made of PEB-4046 modules from Imperix [26] which integrates the two IGBTs (IXYS MMIX1X200N60B3H1) and the DC-bus capacitors $(825 \mu \mathrm{F})$. Two of them (on ports 1 and 2) are actively switched at $f_{\mathrm{sw}}$ while the third one is passive and is used as a rectifier.

The experiments is carried out with resistive loads rated to 1 , 2, 3 and $4 \mathrm{~kW}$. Fig. 10 shows figure of merit for different load conditions, which are in good agreement with the model, i.e., the amplitude of two input currents are proportional to the load and have the ratio $\frac{1}{3}, \frac{2}{3}$ as defined by the components of the resonant tanks in Table I. Then, for the $4 \mathrm{~kW}$ load, different values of $V_{S}$, between $-15 \mathrm{~V}$ and $25 \mathrm{~V}$, are considered in order to show the effects of circulating currents. Fig. 11 shows the measured current waveforms for two different operating points. The powers $P_{1}, P_{2}$ and $P_{3}$ are directly measured at the DC terminals (using a N4L PPA5500 power analyzer). Power analyzer measurements well match calculations computed from

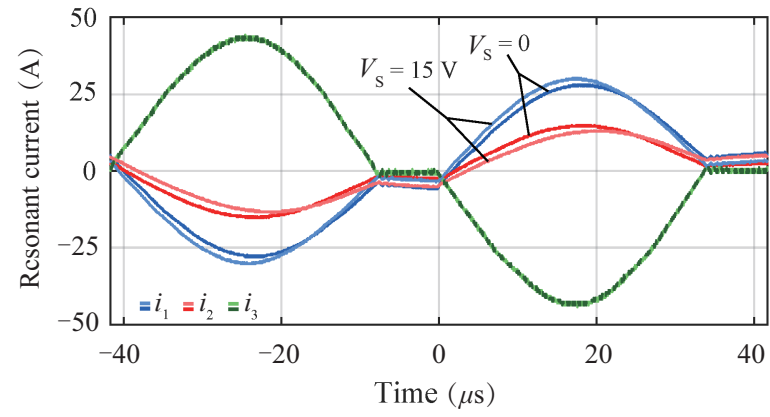

Fig. 11. Resonant current waveforms (oscilloscope import) from measurement at $4 \mathrm{~kW}$. The currents $i_{1}$ and $i_{2}$ are affected by the introduction of differential voltage $V_{\mathrm{S}}$, but the current to the load, namely $i_{3}$ is not affected. To be noted that from $t=-40 \mu \mathrm{s}$ to 0 , the currents flow in $S_{1 L}, S_{2 L}$ and $D_{3 L}$ while from $t=0$ to $40 \mu \mathrm{s}$, the currents currents circulate in $S_{1 H}$ and $S_{2 H}$ and $S_{3 H}$.
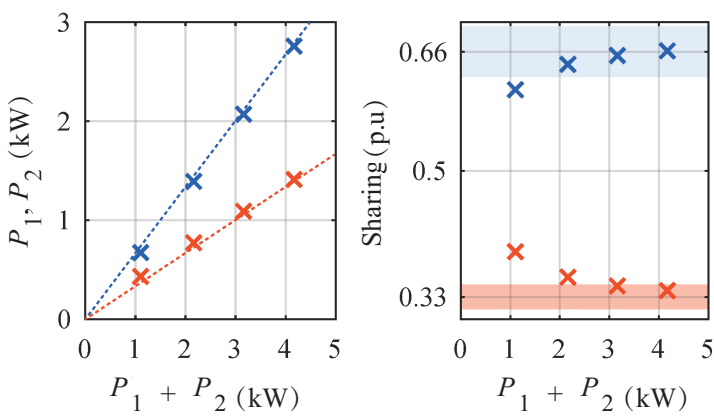

Fig. 12. On the left, powers $\left(P_{1}\right.$ in blue and $P_{2}$ in red) and, on the right, their corresponding sharing part $P_{k} /\left(P_{1}+P_{2}\right)$ when $V_{\mathrm{S}}=0$. Apart from some deviations at light load conditions, the power sharing is constant and reflecting the ratio of the inductance defined in Table I, namely $L_{2} /\left(L_{1}+L_{2}\right)=2 / 3$ and $L_{1} /\left(L_{1}+L_{2}\right)=1 / 3$ (at low power operation, the impact of switching losses, which are load independent and not included in the model, is not negligible).

the time-domain waveforms (acquired by the oscilloscope). $P_{\mathrm{P}}$ and $P_{\mathrm{S}}$ are computed from these latter. Fig. 12 depicts the influence of the tank impedance ratio on the sharing of $P_{\mathrm{P}}$ between $P_{1}$ and $P_{2}$. The natural sharing characteristic is defined by the tank design and is maintained with changing load on the output (third) port. The losses effects on this result are clear: when the output power increases, the weight of losses terms in (32) is smaller and, therefore, (24)-(25) are more accurate. However, when $P_{\mathrm{p}}$ is small, all the terms of (32) have an impact. In order to deep into the accuracy of the model with losses consideration, Figs.13 and 14 are assessed. The effect of $V_{\mathrm{S}}$ on $P_{\mathrm{S}}$ is shown in Fig. 13, illustrating that for the constant output load, the power sharing is highly sensitive to variation of $V_{\mathrm{S}}$. The impact of $V_{\mathrm{S}}$ on the efficiency is depicted in Fig. 14 for the different loads although the lab set-up hardware has not been optimized in that sense, and its purpose is to validate power sharing characteristics.

\section{CONCLusion}

This paper presents the model and the power sharing characteristics for a three-terminal SRC based DC-transformer for SST applications requiring an energy storage functionality. Among the possible modes of operation, the one considering 


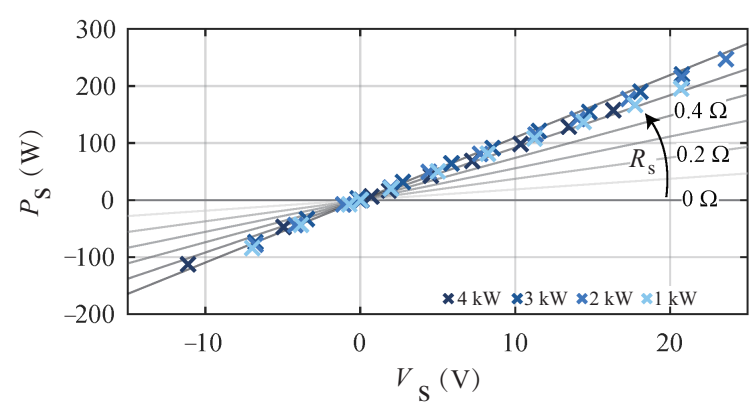

Fig. 13. Measured $P_{\mathrm{S}}$ and effect of $R_{\mathrm{S}}$ on the theoretical curve. For $R_{\mathrm{S}}=0$, it would be zero for any $V_{\mathrm{S}}$. The experiments show that $R_{\mathrm{S}}$, which represent the conduction losses and the switching losses associated to the circulating power flow, is around $0.55 \Omega$.

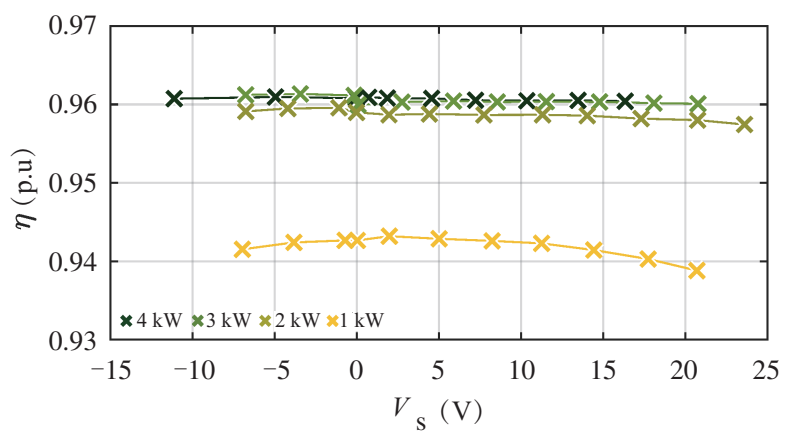

Fig. 14. Efficiency for various operating points. The switching losses depending on the turn off currents $\left(i_{1}(0)\right.$ and $\left.i_{2}(0)\right)$ which are set by $L_{\mathrm{m}}$ and are load independent, the efficiency is reduced for light load conditions. Additional losses appear with the circulation of current $i_{\mathrm{S}}$, which explains the drop of efficiency for increasing $\left|V_{\mathrm{s}}\right|$.

two inputs and one output is the most complex and is therefore analyzed in this paper. In order to get a compact analytic solution, a model based the separation of the power flow into a main power (from the active ports to the load port) and a circulating power (between the active ports) is proposed ("P" and " $\mathrm{S}$ " equivalent circuits). The modelling reveals that the natural power sharing is mostly impacted by the resonant tank design parameters. The sharing is preserved in the absence of differential voltage between the active ports and can be, to some extent, influenced by further manipulation on the differential voltage which introduces a circulating power flow (influenced as well by the losses and parasitic resistances). However, if high circulating currents are forced, the ZVS conditions may be compromised. This gives basic design insights to size the resonant tank inductances in a way to benefit from soft switching in a specified operating region. The experimental results, obtained with a lab-scale prototype, match and effectively verify the proposed model.

\section{REFERENCES}

[1] M. Liserre, G. Buticchi, M. Andresen, G. D. Carne, L. F. Costa, and Z. Zou, "The smart transformer: Impact on the electric grid and technology challenges," IEEE Industrial Electronics Magazine., vol. 10, no. 2, pp. 46-58, Jun. 2016.
[2] F. Briz, M. Lopez, A. Rodriguez, and M. Arias, "Modular power electronic transformers: Modular multilevel converter versus cascadedh-bridge solutions," IEEE Industrial Electronics Magazine, vol. 10, no. 4, pp. 6-19, Dec. 2016.

[3] L. F. Costa, G. D. Carne, G. Buticchi, and M. Liserre, "The smart transformer: A solid-state transformer tailored to provide ancillaryservices to the distribution grid," IEEE Power Electronics Magazine, vol. 4, no. 2, pp. 56-67, Jun. 2017.

[4] J. E. Huber and J. W. Kolar, "Applicability of solid-state transformers intoday's and future distribution grids," IEEE Transactions on Smart Grid, p. 1, 2018.

[5] C. Gu, Z. Zheng, and Y. Li, "A power electronic transformer (PET) withmultiport bidirectional resonant dc-dc converters for electric tractionapplications," in 2015 IEEE Transportation Electrification Conference and Expo (ITEC), June 2015, pp. 1-6.

[6] Z. Pavlović, J. A. Oliver, P. Alou, Ó. Garcia, and J. A. Cobos, "Bidirectional multiple port dc/dc transformer based on a series resonant converter," in 2013 Twenty-Eighth Annual IEEE Applied Power Electronics Conference and Exposition (APEC), Long Beach, CA, 2013, pp. 1075-1082.

[7] K. Tomas-Manez, Z. Zhang, and Z. Ouyang, "Multi-port isolated LLC resonant converter for distributed energy generation with energy storage," in 2017 IEEE Energy Conversion Congress and Exposition (ECCE), Cincinnati, OH, 2017, pp. 2219-2226.

[8] Y. Tran and D. Dujic, "A multiport medium voltage isolated DCDC converter," IECON 2016 - 42nd Annual Conference of the IEEE Industrial Electronics Society, Florence, 2016, pp. 6983-6988.

[9] Y. Tran and D. Dujic, "A multiport isolated DC-DC converter," in 2016 IEEE Applied Power Electronics Conference and Exposition (APEC), Long Beach, CA, 2016, pp. 156-162.

[10] Y. Tran, D. Dujić, and P. Barrade, "Multiport resonant DC-DC converter," in IECON 2015 - 41st Annual Conference of the IEEE Industrial Electronics Society, Yokohama, 2015, pp. 003839-003844.

[11] D. Dujic et al., "Power electronic traction transformer-Low voltageprototype," IEEE Transactions on Power Electronics, vol. 28, no. 12 , pp. 5522-5534, 2013.

[12] C. Zhao et al., "Power electronic traction transformer-Medium voltageprototype," IEEE Transactions on Industrial Electronics, vol. 61, no. 7, pp. 3257-3268, July 2014.

[13] U. Javaid, D. Dujić, and W. van der Merwe, "MVDC marine electrical distribution: Are we ready?," in IECON 2015 - 41st Annual Conference of the IEEE Industrial Electronics Society, Yokohama, 2015, pp. 000823-000828.

[14] Z. Jin, G. Sulligoi, R. Cuzner, L. Meng, J. C. Vasquez, and J. M. Guerrero,"Next-generation shipboard dc power system: Introduction smartgrid and dc microgrid technologies into maritime electrical netowrks," IEEE Electrification Magazine, vol. 4, no. 2, pp. 45-57, June 2016.

[15] H. Choi, M. Ciobotaru, M. Jang, and V. G. Agelidis, "Performance ofmedium-voltage dc-bus pv system architecture utilizing high-gain dc-dcconverter," IEEE Transactions on Sustainable Energy, vol. 6, no. 2, pp.464-473, April 2015.

[16] C. Zhan, C. Smith, A. Crane, A. Bullock, and D. Grieve, "Dc transmissionand distribution system for a large offshore wind farm," in AC and DC Power Transmission, 2010. ACDC. 9th IET International Conference on, Oct. 2010, pp. 1-5.

[17] "Dc for efficiency," ABB Review 4/13, 2013. [Online]. Available: https://library.e.abb.com/public/60289ad65efef903c1257c5a0040a3 7b/ 16-21\%204m412 EN 72dpi.pdf

[18] M. Stieneker and R. W. De Doncker, "Medium-voltage DC distribution grids in urban areas," in 2016 IEEE 7th International Symposium on Power Electronics for Distributed Generation Systems (PEDG), Vancouver, BC, 2016, pp. 1-7.

[19] I. Villar, L. Mir, I. Etxeberria-Otadui, J. Colmenero, X. Agirre, and T. Nieva, "Optimal design and experimental validation of a Medium-Frequency 400kVA power transformer for railway traction applications," 2012 IEEE Energy Conversion Congress and Exposition (ECCE), Raleigh, NC, 2012, pp. 684-690.

[20] M. Mogorovic and D. Dujic, " $100 \mathrm{~kW}, 10 \mathrm{kHz}$ medium frequency transformerdesign optimization and experimental verification," IEEE Transactions onPower Electronics, vol. (in press), 2018. 
[21] C. Oeder and T. Duerbaum, "ZVS investigation of 1lc converters based on FHA assumptions," in 2013 Twenty-Eighth Annual IEEE Applied Power Electronics Conference and Exposition (APEC), Long Beach, CA, 2013, pp. 2643-2648.

[22] M. A. Halim, M. N. Seroji, and M. N. B. Hidayat, "Analysis characteristicsand optimal design procedure of half-bridge LLC loaded resonant converter," in 2012 IEEE Int. Conf. Power and Energy (PECon), Dec. 2012, pp. 564-569.

[23] D. Rothmund, J. E. Huber, and J. W. Kolar, "Operating behavior and design of the half-cycle discontinuous-conduction-mode seriesresonant-converter with small DC link capacitors," in 2013 IEEE 14th Workshop on Control and Modeling for Power Electronics (COMPEL), Salt Lake City, UT, 2013, pp. 1-9.

[24] A. Vidal et al., "A Method for Identification of the Equivalent Inductance and Resistance in the Plant Model of Current-Controlled Grid-Tied Converters," in IEEE Transactions on Power Electronics, vol. 30 , no. 12 , pp. $7245-7261$, Dec. 2015.

[25] C. Adragna, S. De Simone, and C. Spini, "A design methodology for LLCresonant converters based on inspection of resonant tank currents," in 2008 Twenty-Third Annual IEEE Applied Power Electronics Conferenceand Exposition, Feb 2008, pp. 1361-1367.

[26] “Imperix Ltd," [Online]. Available: https://imperix.ch/products/power/ peb.

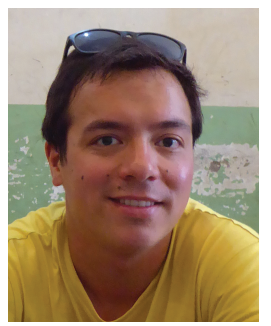

Yan-Kim Tran received the B.Sc. and M.Sc. degree in electrical engineering from the École Polytechnique Fédérale de Lausanne (EPFL), Lausanne, Switzerland, in 2010 and 2012, respectively. From October 2014, he is with the Power Electronics Laboratory as a doctoral assistant. His research interests include the design and control of converters for medium voltage applications.

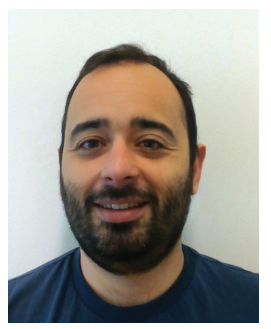

Francisco D. Freijedo received the M.Sc. degree in physics from the University of Santiago de Compostela, Santiago de Compostela, Spain, in 2002 and the Ph.D. degree in Electrical Engineering from the University of Vigo, Vigo, Spain, in 2009. From 2005 to 2011, he was a Lecturer in the Department of Electronics Technology, University of Vigo. From 2011 to 2014, he worked in Gamesa Innovation and Technology as a Power Electronics Control Engineer, where he was involved in Wind Energy projects. From 2014 to 2016, he was a Postdoctoral Researcher in the Department of Energy Technology, Aalborg University. Since 2016, he is a Scientific Collaborator of the Power Electronics Laboratory, École Polytechnique Fédérale de Lausanne. His research interests include many power conversion technologies and challenging control problems.

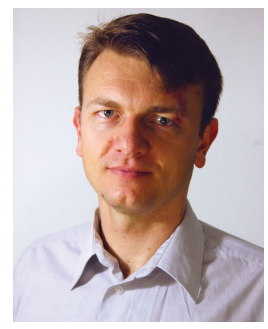

Drazen Dujic received the Dipl. -Ing. and M.Sc. degrees from the University of Novi Sad, Novi Sad, Serbia, in 2002 and 2005, respectively, and the Ph.D. degree from the Liverpool John Moores University, Liverpool, U.K., in 2008, all in electrical engineering. From 2002 to 2006, he was with the Department of Electrical Engineering, University of Novi Sad as a Research Assistant, from 2006 to 2009 with Liverpool John Moores University as a Research Associate. From 2009 till 2013, he was with ABB Corporate Research Centre, Switzerland, as a Principal Scientist working on the power electronics projects spanning the range from low-voltage/power SMPS in below kilowatt range to medium voltage high-power converters in a megawatt range. From 2013 till 2014, he was with ABB Medium Voltage Drives, Turgi, Switzerland, as R\&D Platform Manager, responsible for ABB's largest IGCT based medium voltage drive ACS6000. He is currently with École Polytechnique Fédérale de Lausanne EPFL, Lausanne, Switzerland, as an Assistant Professor and the Director of the Power Electronics Laboratory. His current research interests include the areas of design and control of advanced high-power electronics systems and high performance drives. He has authored or coauthored more than 100 scientific publications and has filed twelve patents. He is an Associate Editor for IEEE Transactions on Industrial Electronics, IEEE Transactions on Power Electronics and IET Electric Power Applications. He has received the First Prize Paper Award by the Electric Machines Committee of the IEEE Industrial Electronics Society at IECON-2007. In 2018 he has received EPE Outstanding Service Award and in 2014 the Isao Takahashi Power Electronics Award for outstanding achievement in power electronics. 